\title{
An Assessment of Dog Behaviour with Regard to Scientific Research Findings
}

\author{
Paweł Bury-Burzymski ${ }^{1}$ and Marta Walczak ${ }^{2 *}$ \\ 1Department of Animal Sciences, Warsaw University of Life Sciences, Poland \\ ${ }^{2}$ Department of Animal Behavior, Institute of Genetics and Animal Breeding PAS \\ Jastrzębiec, Poland
}

\section{Review Article \\ Volume 2 Issue 2}

Received Date: February 22, 2017

Published Date: March 02, 2017

*Corresponding author: Marta Walczak, Department of Animal Behavior, Institute of Genetics and Animal Breeding PAS Jastrzębiec, ul. Postępu 36A 05-552 Magdalenka, Poland, E-mail: martawalczak@yahoo.com

\section{Abstract}

Dogs have been used in the field of law enforcement for over 100 years. Despite advances in the overall performance of police officers due to improved training methods and use of various innovative devices, it is hard to imagine a fully effective police force operating without the involvement of specialist dog-handler teams. The uniformed services generally hold in high regard the role that a well-trained canine team can play, serving as an essential deterrent to criminal activity, as well as assisting police departments in the locating of illegal drugs and explosives, in tracking fugitives and with finding missing persons or locating bodies. An understanding of general dog behaviour and interaction with different environments are essential elements, not only in the case of assessing candidate suitability for different types of training programme but also for behaviour evaluation of a companion animal. The everincreasing demand for both working and pet dogs has seen the study of dog behaviour find itself a niche position in the realm of scientific research.

Keywords: Dogs Behavior; Behavioral Testing; Working Dogs

\section{Temperament and Personality}

In the early 1920s the Nobel Prize laureate Ivan Pavlov began his research aimed at identifying the basis of dog temperament. Yet despite the successful launch of such research on animal temperament and personality, this particular field never evolved as a separate branch of psychology, as occurred in the case of human psychology [1]. Two main terms in ethological literature exist: temperament and personality. These terms seem to be indistinguishable, which is caused by a different interpretation of the meaning of the two concepts. In the field of human psychology, temperament is defined as biologically determined. Its features may be identified in early childhood and are considered to be the foundation of personality; in turn, personality is understood as a product of the social environment and is shaped in later periods of development [1]. Temperament is sometimes considered to be those behavioural traits which are likely to be repeated under similar circumstances [2]. These definitions have not been universally accepted by researchers in the field of human psychology though and scientists dealing with animals are in even less agreement in this respect [3]. Across the non-human animal personality literature, "personality" and 'temperament" and indeed "temperament" and "character" are often used interchangeably, despite having clear and separate historical definitions in human psychology $[3,1,4]$. The largest study of dog personality involved more than 15 thousand animals from164 breeds and was carried out by Svartberg and Forkman [5] using the dog mentality assessment (DMA) used by the Swedish Working Dog Association. The study consisted of each dog facing 10 trials which included the sudden appearance of a dummy, a gunshot, 


\section{Open Access Journal of Veterinary Science \& Research}

a metallic sound and two persons dressed in white sheets. Exhibited reactions of participant animals revealed five dimensions identified as "Playfulness", "Curiosity/Fearlessness", "Chase proneness", "Sociability" and "Aggressiveness". Further investigation revealed that the first three characteristics are closely linked to one another, creating one "dimension" of personality which is similar to "shyness boldness" previously identified in both humans and animals, and to human "supertraits" (a combination of Extraversion and Neuroticism). The dog is a species whose distinct personalities are very clear/visible, a quality also observed in humans. The Five-Factor Model (FFM) of personality is the most broadly accepted and applied model of personality in the field of human trait psychology. The FFM explains personality as an interaction of the traits of "extraversion", "agreeableness", "conscientiousness", "neuroticism" and "openness to experience". Based on research of dogs' personality and that of their owners, Gosling and John [3] found that the basic types found in human FFM have their equivalents in dogs. For example, neuroticism in humans corresponds to emotional reactivity in dogs, human agreeableness is analogous to affection in dogs, human "openness and conscientiousness" is reflected in dogs learning and obedience ability.

\section{Behavioral Phenotyping Methods}

A meta analysis conducted by Jones and Gosling [1] of 51 scientific research projects undertaken on dogs showed that those traits most widely studied are timidity, reactivity, susceptibility to training, sociability, aggressiveness, subordination and domination, and activity. Most programmes aimed at working dogs do not evaluate individual behaviour in a specific situation/test but rather seek to identify a number of features at the same time as describing the dog. Often these measures are subjected to factor or principal component analysis (PCA), which are data reduction techniques that statistically identify consistently correlated measures within a data set and place them into factors [6]. The composition of these factors can be used to describe the various behavioral traits exposed by the test and can also be used to predict a dog's behavior in other, similar future situations [7].

There are four main methods used in the assessment of dog behaviour: test batteries, questionnaire based surveys, an expert-ratings approach and observational tests. The most common method employed in assessing behaviour is that of test batteries, where dogs are judged in response to specific stimuli, usually previously unknown to the dog, and which can trigger a specific behaviour (eg. fear or aggression). In theory, the battery testing method is the most effective in achieving an objective assessment of the animal's behaviour due to the stability and uniformity of conditions used in carrying out the procedure. This method is used for example by the uniformed services in order to assess the suitability of dogs for various purposes [7] and also typically in order to evaluate anxiety and aggression levels in shelter dogs to determine their suitability for re-homing, helping to increase the rate of successful adoptions [8]. Two main components may be distinguished in the battery of tests: the stimulus used in the procedure and classification of response to particular stimulus [7].

Test batteries were used by Haverbeke et al. [9] in response to behavioural problems exhibited by military dogs whilst serving in the Belgian Army, problems that included low obedience during exercises and a high rate of biting incidents. The testing procedure consisted of socialisation trials carried out in a variety of simulated situations (petting of dog with artificial hand by unknown person, neutral approach of unknown persons, reaction to opened umbrella or flapped blanket, reaction to child-like doll and remote controlled toy car, auditory stimulus) in order to detect aggressive or fearful dogs. Particular behaviours relating to aggression, such as barking, growling, grinning, snapping of teeth, attempts to avoid a stimulus and body posture were then evaluated.

Questionnaire-based surveys have also commonly been used to assess canine behavioural attributes. They have the benefit of being able to be directed to large population samples and allow the long-term evaluation of the behaviour of a test subject in various situations. When questionnaires are prepared, special attention should be paid to the form and structure they take in order to ensure correct evaluation of specific characteristics [10]. At present, the most frequently used questionnaire is the Canine Behaviour Assessment Questionnaire (C-BRQ) based on data from over 2000 dogs representing more than 100 breeds [11]. Duffy and Serpell [12] Found C-BRQ to be a useful tool in selecting appropriate candidate dogs at the age of 6 months as guides for the blind. Less commonly employed is the expert-rating approach, whereby veterinarians or others with recognized expertise rate breeds, as opposed to individual dogs, for specific traits [13-15]. The last of the four major approaches is the observational test, which relies on expert observation of individual dogs under natural circumstances, such as during video-recorded walks [16].

Two distinct methodologies are relied upon for measuring personality: behavioural codings and behavioural ratings $[17,18]$. Behavioural codings typically attempt to measure observed, discrete behaviours, often generated from an ethogram, such as the frequency and duration of a particular posture. For 
example, one study used the number of lines (marked on the floor of a test arena) crossed by individual dogs as an indicator of locomotor activity [19]. Ratings typically consist of broader judgments regarding a dog's standing on a behavioural trait made by people familiar with the dog. For example, in one study dogs were rated on a 1-5 Like rt scale according to their playfulness with a rag in a standardised test [5].

\section{Criteria for Behavioural Measurement}

In order for the results of a particular behavioural assessment to be valuable it must fulfill three scientific criteria: reliability, validity and predictive value [20]. For the test to be reliable and valid, it must be standardised. This requires the procedure to be clearly described and followed so that it can be consistently repeated. When an evaluation is optimally standardised, the only remaining variable is the response of the dog $[2,12]$. Reliability rests on the reproducibility of measurements and the degree to which test scores can be said to be free from errors of measurement, something which may be achieved by inter-rater agreement, intra-rater agreement and by test-retest correlation $[1,2,20]$. For a measuring tool to be deemed appropriate, a high reliability value of agreement of $>0.7$ should exist. Inter-observer reliability measures the likelihood that different observers assessing the same dog on the same occasion will get the same results. In the few published studies of working dogs, the results of inter-rater correlations are mixed and vary from no agreement to almost unanimous agreement [7]. For example, in a study of companion dogs by Ley et al. [21], five aggregate traits were not high with an overall average of 0.62 . In contrast, a study by Goddard and Beilharz [22] found inter-rater agreement for single behavioural traits ranged from 0.000 for "willingness to carry out commands" to 0.7 for "nervousness of people, traffic and strange places". In turn, Sinn et al [7] found that in applying a 15 item behavioural instrument over 3 sessions, inter-rater agreement was high (0.86). For such behavioural traits as "sensitivity to sounds", "frontal bite" and "chase attack" though, the inter-rater agreement was low when evaluated for first measurement time $(0.30,0.40$, and 0.27 respectively). Intra-rater reliability measures the consistency of the single observer over time. Due to the fact that a dog's behaviour may change over an extended period, it is advised that intra-observer reliability be assessed through the use of video recording equipment, thus allowing results to be compared [20]. Test-retest reliability examines result correlation between two tests separated by a certain period of time where there can be confidence that testing parameters remain consistent [1]. Achieving reliability by re-testing can be challenging, especially in the case of the testing of reactions to novel stimulus, as repeat testing with previously known situations or stimuli may result in habituation $[8,23]$. According to Svartberg et al. [23] behaviours relating to the concept of temperament should remain constant between intervals. Researchers list a number of factors to help explain why behaviour between the first and subsequent testing may vary, including the current psychophysical state of the subject animal and its hunger or disease state. Nevertheless, Jones and Gosling Jones and Gosling [1] highlight the value of results obtained by means of subsequent testing when an understanding of particular testing conditions exists. For example, Walczak et al. [24] evaluated fear and aggression in police candidate dogs over two intervals of time. Forty-three German Shepherds were given four socialisation tests: the approach of 3 unfamiliar people at normal walking speed looking away from the dog, the neutral approach of 3 unfamiliar people staring at the dog, the approach of an unfamiliar man, and approach of an unfamiliar woman staring directly at the dog. The dogs were subsequently ranked according to body posture, aggressive behaviour, avoiding the stimulus and stress related behaviours. The study demonstrated that training for patrol purposes either reduced or had a negligible effect on the level of fearful-aggressive behaviour in $53.5 \%$ of dogs while increasing the level of fear-related aggression in $46.5 \%$ of dogs $(\mathrm{p}<0.01)$. The results suggest that training for patrol purposes with a particular focus on defense exercises in the last month of training could increase fear-related aggression in certain dogs [24]. Netto and Planta [9] have suggested that dogs may react more aggressively in some repeated situations because they may have perceived that they "won" last time (eg: they were rewarded for avoiding a potential threat on the first occasion.

The last and ultimately most important criterion for judging the usefulness of tools employed to assess dog behaviour is that of their predictive value. Such a tool should have the capacity to accurately determine future behaviour of dogs facing similar challenges. The predictive value of behavioural tests differs among different studies and depends on the particular behavioural trait under evaluation. A study by Rooney and Bradshaw [25] on 244 handlers from 6 different units in Great Britain found that "the use of smell alone while working", "stamina", "learning for reward" and "distraction while working" are traits which can predict success in sniffing dogs. In turn, Maejima et al. [26] emphasise that features such as "activity", "obedience", "concentration", "fearfulness" and "object interest" are traits that can be evaluated to accurately identify successful sniffing dogs. Wilsson and Sundgren [27] reported poor correspondence between puppy test results and adult dog behaviour and performance for service dogs in a sample of 630 German shepherd dogs. Similarly, Asher et al. [28] followed up 465 dogs 


\section{Open Access Journal of Veterinary Science \& Research}

assessed in a puppy test and subsequently trained as guide dogs and found low predictability of successful certification. Of the 450 dogs that scored above the proposed cut-off point in the behavioural test, $66 \%$ reached certification, compared to $64 \%$ in the complete sample. In contrast to success, failure was more accurately predicted by the test, as 14 of the 15 dogs that scored below the cut-off point did not reach certification [28]. The lack of success in predicting adult behaviour from information gained in a number of these puppy tests could be explained by continuing neural and behavioural changes that occur within juvenile dogs and which are likely to continue past sexual maturity, stabilising only at social maturity [29]. This view is supported by evidence which shows that the predictive ability of behavioural tests improve as an animal ages [22,30-32,27].

\section{Conclusions}

Understanding behaviour variation among dogs and how it refers to their predisposition to a particular type of work remains an unresolved issue for breeding organizations and their programmers' for working dogs. Unfortunately, no fully validated method for predicting the behaviour of an evaluated animal yet exists. Use of the best currently available behavioral testing or attempts at improving existing methods have significant economic justification. The total cost of dog purchase, accommodation and training is estimated at approx. 18,500 USD, meaning that even small improvements in certification outcome prediction could result in substantial potential savings. Alongside economic considerations for performing reliable assessments of dog behaviour, there are other pressing issues including the amount of time invested in training future service dogs as well as the problem of finding an alternative owner in the case of their withdrawal from training, as an adult individual, at the age of 2 to 3 years, is not such an attractive animal for a potential buyer as a young dog or a puppy.

\section{Acknowledgements}

The review is supported by NCN grant Sonata \#2012/07/D/NZ9/03370 and is a part of Master Thesis of P.B.-B.

\section{References}

1. Jones AC, Gosling SD (2005) Temperament and personality in dogs (Canis familiaris): A review and evaluation of past research. Applied Animal Behaviour Science 95(1): 1-53.

2. Diederich C, Giffroy JM (2006) Behavioural testing in dogs: a review of methodology in search for standardisation. Applied Animal Behaviour Science 97(1): 51-72.

3. Gosling SD, John OP (1999) Personality dimensions in nonhuman animals: a cross-species review. Current directions in psychological science 8(3): 69-75.

4. Ruefenacht S, Gebhardt-Henrich S, Miyake T, Gaillard C (2002) A behaviour test on German Shepherd dogs: heritability of seven different traits. Applied Animal Behaviour Science 79(2): 113-132.

5. Svartberg K, Forkman B (2002) Personality traits in the domestic dog (Canis familiaris). Applied Animal Behaviour Science 79(2): 133-155.

6. Goodloe LP, Borchelt PL (1998) Companion dog temperament traits. Journal of Applied Animal Welfare Science 1(4): 303-338.

7. Sinn DL, Gosling SD, Hilliard S (2010) Personality and performance in military working dogs: Reliability and predictive validity of behavioral tests. Applied Animal Behaviour Science 127(1): 51-65.

8. Netto WJ, Planta DJ (1997) Behavioural testing for aggression in the domestic dog. Applied Animal Behaviour Science 52(3-4): 243-263.

9. Haverbeke A, De Smet A, Depiereux E, Giffroy JM, Diederich C (2009) Assessing undesired aggression in military working dogs. Applied Animal Behaviour Science 117(1): 55-62.

10. Hall NJ, Wynne CD (2012) The canid genome: behavioral geneticists' best friend? Genes, Brain and Behavior 11(8): 889-902.

11. Foyer P, Bjällerhag N, Wilsson E, Jensen P (2014) Behaviour and experiences of dogs during the first year of life predict the outcome in a later temperament test. Applied Animal Behaviour Science 155: 93-100.

12. Duffy DL, Serpell JA (2012) Predictive validity of a method for evaluating temperament in young guide and service dogs. Applied Animal Behaviour Science 138(1): 99-109.

13. Hart B, Hart L (2005) Breed-specific profiles of canine (Canis familiaris) behavior. Current Issues and Research in Veterinary Behavioral Medicine 107-112.

14. Hart BL (1995) Analysing breed and gender differences in behaviour. The domestic dog: Its 


\section{Open Access Journal of Veterinary Science \& Research}

evolution, behaviour and interactions with people 65-77.

15. Hart BL, Hart LA (1985) Selecting pet dogs on the basis of cluster analysis of breed behavior profiles and gender. Journal of the American Veterinary Medical Association 186(11): 1181-1185.

16. Murphy JA (1995) Assessment of the temperament of potential guide dogs. Anthrozoös 8(4): 224-228.

17. Gosling SD (2001) From mice to men: what can we learn about personality from animal research? Psychological bulletin 127(1): 45-86.

18. McGarrity ME, Sinn DL, Thomas SG, Marti CN, Gosling SD (2016) Comparing the predictive validity of behavioral codings and behavioral ratings in a working-dog breeding program. Applied Animal Behaviour Science 179: 82-94.

19. Hennessy MB, Voith VL, Mazzei SJ, Buttram J, Miller DD, et al. (2001) Behavior and cortisol levels of dogs in a public animal shelter, and an exploration of the ability of these measures to predict problem behavior after adoption. Applied Animal Behaviour Science 73(3): 217-233.

20. Taylor KD, Mills DS (2006) The development and assessment of temperament tests for adult companion dogs. Journal of Veterinary Behavior: Clinical Applications and Research 1(3): 94-108.

21. Ley JM, McGreevy P, Bennett PC (2009) Inter-rater and test-retest reliability of the Monash Canine Personality Questionnaire-Revised (MCPQ-R). Applied Animal Behaviour Science 119(1): 85-90.

22. Goddard M, Beilharz R (1983) Genetics of traits which determine the suitability of dogs as guidedogs for the blind. Applied Animal Ethology 9(3-4): 299-315.

23. Svartberg K, Tapper I, Temrin H, Radesäter T, Thorman S (2005) Consistency of personality traits in dogs. Animal Behaviour 69(2): 283-291.

24. Walczak M, Adamkiewicz E, Walasek A, Lisowski P, Jezierski $\mathrm{T}$ (2014) Evaluation of fear-related aggression in police patrol dogs to unfamiliar humans during socialization stimulus. Journal of Veterinary Behavior: Clinical Applications and Research 9(6): e13.
25. Rooney NJ, Bradshaw JW (2004) Breed and sex differences in the behavioural attributes of specialist search dogs-a questionnaire survey of trainers and handlers. Applied Animal Behaviour Science 86(1): 123-135.

26. Maejima $M$, Inoue-Murayama $M$, Tonosaki $K$, Matsuura N, Kato S, et al. (2007) Traits and genotypes may predict the successful training of drug detection dogs. Applied Animal Behaviour Science 107(3): 287-298.

27. Wilsson E, Sundgren PE (1998) Behaviour test for eight-week old puppies-heritabilities of tested behaviour traits and its correspondence to later behaviour. Applied Animal Behaviour Science 58(1): 151-162.

28. Asher L, Blythe S, Roberts R, Toothill L, Craigon PJ, et al. (2013) A standardized behavior test for potential guide dog puppies: Methods and association with subsequent success in guide dog training. Journal of Veterinary Behavior: Clinical Applications and Research 8(6): 431-438.

29. Overall K (2013) Manual of clinical behavioral medicine for dogs and cats $1^{\text {st }}$ (Edn.) Elsevier Health Sciences.

30. Fratkin JL, Sinn DL, Patall EA, Gosling SD (2013) Personality consistency in dogs: a meta-analysis. PLoS One 8(1): e54907.

31. Goddard M, Beilharz R (1986) Early prediction of adult behaviour in potential guide dogs. Applied Animal Behaviour Science 15(3): 247-260.

32. Harvey ND, Craigon PJ, Sommerville R, McMillan C, Green M, et al. (2016) Test-retest reliability and predictive validity of a juvenile guide dog behavior test. Journal of Veterinary Behavior: Clinical Applications and Research 11: 65-76. 